\title{
Umweltschäden durch Heizöl nach Hochwasserereignissen
}

\author{
Ursula Stephan $\cdot$ Detlef Deininger $\cdot$ Johannes Kardos $\cdot$ Ute Strobel
}

Erhalten: 27. August 2008/Akzeptiert: 22. März 2009/Online veröffentlicht: 3. September 2009

(C) Springer-Verlag 2009

Zusammenfassung Hintergrund Durch hohe Sicherheitsvorkehrungen bei Herstellung, Transport und Lagerung von Heizöl kommt es kaum noch vor, dass Heizöl in die Umwelt freigesetzt wird. Eine Ausnahme von dieser Erfahrung bildeten die mit dem „Jahrhunderthochwasser“ 2002 in Deutschland verbundenen Heizölaustritte aus Heizöltanks vor allem im privaten Bereich.

In einer im Auftrag von IWO (Institut für wirtschaftliche Oelheizung e.V.) erstellten Studie haben die Autoren das Umweltverhalten von Heizöl nach Hochwasserereignissen untersucht.

Ziel Da sich Heizöl nach seiner Freisetzung rasch auf Wasseroberflächen ausbreitet und große Flächen kontaminiert, wird ein großer Umweltschaden erwartet. Zur Bewertung muss das Verhalten von Heizöl in Wasser und Böden ebenso wie die Wirkung von Heizöl auf höhere und niedere Tiere und Pflanzen, auf Wasserorganismen sowie auf die Flora und Fauna des Bodens differenziert betrachtet werden. Aus der Bewertung der Umweltgefahren können erforderliche behördliche Maßnahmen sowie Vorsorge und Sicherungsmaßnahmen gegen die Gefahren durch Hersteller und Nutzer von privaten Heizölanlagen abgeleitet werden.

Methodisches Vorgehen Zur Beantwortung der unterschiedlichen Fragestellungen stützte sich das Autorenteam auf die umfangreichen Analysen des Sondermesspro-

Verantwortlicher Herausgeber: Jens Didszun

D. Deininger $(\bowtie) \cdot J$. Kardos

Institut für innovative Technologien ITA GmbH,

Hubertus 1a, 06366 Köthen, Deutschland

E-Mail: ita.koethen@t-online.de

U. Stephan · U. Strobel

Gefahrstoff-Büro Prof. Stephan und Dr. Ute Strobel GbR,

Heidehäuser 6, 06120 Halle, Deutschland

E-Mail: Ute.Strobel.Gefahrstoffbuero@t-online.de gramms Sachsen-Anhalt, auf Befragungen von Betroffenen (Bevölkerung sowie Behörden), auf Luftbildaufnahmen, auf umfangreiche Literaturbefunde einschließlich ökotoxikologischer Untersuchungen, auf Erfahrungen bei der über 70-jährigen Verwendung von Heizöl im Pflanzenschutz und auf praktische Erfahrungen bei der großräumigen Sanierung von Ölschäden nach Havarien bzw. Unfällen. Die Autoren nahmen Bilanzierungen auf der Basis der vorhandenen Analysenergebnisse und eigener Berechnungen vor.

Ergebnisse Die Freisetzung von Heizöl in die Luft stellt kein besonderes Problem dar, da es zu ca. $40 \%$ schnell verdunstet und vollständig zu Kohlendioxid und Wasser abgebaut wird. Charakteristisch ist neben der Verdunstung die rasche Ausbreitung des Heizöls auf der Wasseroberfläche zu sehr dünnen Schichten. Bei den typischen, bunt schillernden Öllachen hat sich z. B. ein Kubikmeter Öl auf einer Wasserfläche von ca. $3 \mathrm{~km}^{2}$ ausgebreitet, das entspricht einer Ölmenge von $3 \mathrm{ml} / \mathrm{m}^{2}$ Fläche, die nach dem Abtrocknen im Boden zu einer Ölkonzentration von ca. $3 \mu \mathrm{g} / \mathrm{kg}$ Boden führt und damit um Größenordnungen unter dem natürlichen Gehalt an Kohlenwasserstoffen in Böden liegt. Aufgrund des Absorptionsvermögens der Böden und des mikrobiellen Abbaus im Boden durch ubiquitär verbreitete Kohlenwasserstoff abbauende Mikroorganismen dringt das Öl nur wenige Zentimeter in den Boden ein und wird in wenigen Monaten abgebaut, sodass Grundwasserschäden durch Heizölaustritte nach Hochwasserereignissen praktisch ausgeschlossen sind. Auch beim Hochwasser 2002 wurden bei Nachmessungen wenige Monate nach den Heizölaustritten keine Ölkomponenten mehr im Boden nachgewiesen.

Diskussion Akute Schäden durch Heizöl an der Mikroflora und -fauna des Bodens sowie des Wassers können nicht von vornherein ausgeschlossen werden. So liegen die Schwellenwerte der Schadwirkung für einige Wasserorganismen unterhalb von $1 \mathrm{mg} / \mathrm{l}$. Jedoch wurden diese Werte, 
wie das Sondermessprogramm zum Hochwasser 2002 in Sachsen-Anhalt zeigte, meist weit unterschritten. Detailliert werden in der Studie (Deininger et al. 2006a) Ausbreitung und Verdunstung von Heizöl auf Wasseroberflächen, die Verteilung, die Ausbreitung und der Abbau von Heizöl im Boden beschrieben. Eine quantitative Auswertung des Heizölverbleibs erfolgt anhand eines konkreten Hochwasserereignisses.

Schlussfolgerungen Besondere Maßnahmen an Böden nach Heizölfreisetzungen bei Hochwasserereignissen sind nicht erforderlich. Selbst bei der Sanierung von Mineralölschäden nach Transportunfällen bzw. an Altstandorten mit wesentlich höheren Konzentrationen an Mineralölkohlenwasserstoffen als bei Heizölfreisetzungen nach Hochwasserereignissen wurden mit bodenpflegerischen Maßnahmen durch die natürlichen Mikroorganismen hohe Abbauleistungen erreicht und in wenigen Monaten die Konzentrationen an Kohlenwasserstoffen um 70-90\% reduziert.

Ausblick Mit den vorliegenden Ergebnissen erfolgt eine realistische Bewertung der Umweltgefahren von Heizölaustritten nach Hochwasserereignissen. Daraus können adäquate Maßnahmen für behördliches Handeln und vorsorgende sowie sichernde Maßnahmen für Heizölanlagen in hochwassergefährdeten Gebieten abgeleitet werden.

Schlüsselwörter Heizöl auf Wasseroberflächen · Heizöl in Böden · Heizöl und Ökotoxikologie · Heizölaustritt · Hochwasser · Umweltschäden durch Heizöl · Verdunstung von Heizöl

\section{Environmental harm by fuel oil after flood incidents}

Abstract Background Due to high safety measures in production, transport and storage of fuel oil it rarely occurs, that fuel oil will be released in the environment. One exception of this experience was the fuel oil releases of private fuel oil tanks during the "century flood" 2002 in Germany. By order of IWO (Institut für wirtschaftliche Oelheizung e.V.), the authors investigated the environmental behaviour of fuel oil after flood incidents.

Aim Due to the fast spreading of the fuel oil on water surfaces and the contamination of huge areas one expects large environmental harm. For appraisal the behaviour of fuel oil in water and soil must be studied in detail as well as the effect on high and low developed animals and plants, on water organisms and on the flora and fauna of soil. From the valuation of the environmental harm official measures and measures of precaution and safety by manufacturer and user of private fuel oil installations can be derived.

Main features For considering the various aspects the authors studied the extensive analyses of the special measuring programme of Saxony-Anhalt, used interviews of con- cerned persons (private persons and officials), aerial photos, extensive study of literature including eco-toxicological investigations, experiences of more than 70-years applications of fuel oil in plant protection and practical experiences at large field redevelopment of oil damages following averages and accidents. The authors valuated on the base of results of analyses and on own calculations.

Results The release of fuel oil in the air is no particular problem because about $40 \%$ of the oil fast evaporate and will be decomposed to carbon dioxide and water. In addition to the evaporation a characteristic behaviour is fast spreading of the fuel oil on the water surface to very thin layers. For a typical coloured oil layer e. g. one cubic meter of oil is spread on a water surface of about $3 \mathrm{~km}^{2}$, this corresponds to $3 \mathrm{ml} / \mathrm{m}^{2}$ surface and contaminates the soil after drying up with about $3 \mu \mathrm{g} / \mathrm{kg}$ soil some orders less than the natural content of hydrocarbons in soil. Because of the absorption capacity of soil and the microbial decomposition by everywhere existing hydrocarbons decomposing microorganisms the oil infiltrates only a few centimetre and will be decomposed in a few months, so that ground water detriments not arise practically. By measurements a few months after fuel oil release in the flood 2002 oil components in the soil could not be detected.

Discussion Acute injuries of micro-flora and -fauna in soil and water by fuel oil cannot be excluded from the first. Thus the limiting values of injuries for some water organisms are below $1 \mathrm{mg} / 1$. According to the special measuring programme at the flood 2002 in Saxony-Anhalt however the measured values were mostly wide below of this limit. In detail the spreading and evaporation of fuel oil on water surfaces, the propagation and decomposition of fuel oil in soil will be described. A quantitative valuation of fuel oil distribution in a real flood incident will be given.

Conclusions Particular measures of redevelopment of soil for fuel oil release after flood incidents are not necessary normally. Even at the redevelopment after transport damages or at devastated sites with essential higher oil contaminations of soil in comparison to fuel oil release after flood incidents high decomposition rates are obtained by normal soil improving measures supporting the natural micro-organisms in reducing the hydrocarbon concentration for $70-90 \%$ after a few months.

Perspectives With the described results a realistic valuation of the environmental harm of fuel oil release after flood incidents could be given. From this qualified measures can be derived for official decisions and precautionary and reliable activities at fuel oil installations of flood endangered areas.

Keywords Environmental harm by fuel oil - Evaporation of fuel oil · Flood · Fuel oil and eco-toxicology · Fuel oil in soil · Fuel oil on water surfaces $\cdot$ Fuel oil release 


\section{Aufgabenstellung}

Dank hoher Sicherheitsstandards und einer Vielzahl von Sicherheitsvorkehrungen beim Umgang mit Heizöl (Herstellung, Transport, Lagerung sowie Verwendung) kommt es erfreulicherweise immer seltener vor, dass Heizöl unbeabsichtigt in die Umweltmedien Luft, Wasser und/oder Boden eintreten kann. Die Heizölaustritte beim Hochwasser 2002 wurden hauptsächlich durch den privaten Bereich, vor allem durch unsachgemäße Installation der Heizöltanks, verursacht. Während die Freisetzung von Heizöl in die Luft keine besonderen Probleme bereitet, da es schnell und vollständig zu Kohlendioxid $\left(\mathrm{CO}_{2}\right)$ und Wasser abgebaut wird, ist das Verhalten von Heizöl in Wasser und Boden doch etwas differenzierter zu betrachten. Auch kann die Wirkung von Heizöl auf höhere Tiere und Pflanzen, auf Wasserorganismen sowie auf die Flora und Fauna des Bodens nicht pauschal eingeschätzt werden. Die Diskussion über die Kontamination von Oberflächen und von Grundwasser spielt vor allem auch nach der Freisetzung von Ölen und hier insbesondere von Heizölaustritten bei Hochwasser eine Rolle. Meist bleibt jedoch unbekannt, wie sich das ausgetretene Heizöl verhält, wo es selbst verbleibt und welche Umweltschäden es kurz- oder langfristig bewirkt. Es war Aufgabe der vorliegenden Studie (Deininger et al. 2006a), die Wirkungen von freigesetztem Heizöl nach Hochwasserereignissen zu beschreiben und zu bewerten.

\section{Methodisches Vorgehen}

In der Studie (Deininger et al. 2006a) werden ausgeprägte Hochwasserereignisse, insbesondere das Jahrhunderthochwasser 2002 an Elbe und Donau, beschrieben. Der Auswertung liegen die offiziellen Berichte der Landesämter bzw. Staatsministerien und der damit befassten Behörden zugrunde, mit dem Schwerpunkt von Heizölfreisetzungen sowie dadurch bedingte Beeinträchtigungen der Umwelt. Das Umweltverhalten von Heizöl unter besonderer Berücksichtigung der Umweltmedien Wasser und Boden wird auf der Grundlage eines umfangreichen Literaturstudiums detailliert beschrieben. Dabei wird von den physikalisch-chemischen Eigenschaften von Heizöl sowie vom natürlichen Vorkommen und dem unerwünschten Eintrag in die Umwelt ausgegangen. Im Einzelnen werden das Verhalten von Heizöl und insbesondere die Umwandlungsprozesse bei der Verteilung von Heizöl in Wasser und Boden beschrieben und bewertet. Die ökotoxikologische Wirkung von Heizöl in der Umwelt ist eingehend dargestellt. Grenzwerte zur Bewertung und Ableitung von Maßnahmen werden diskutiert. In Kapitel 4 wird eine praxisbezogene Bewertung zu Verteilung und Abbau von Heizöl in der Umwelt bei Freisetzung nach Hochwasserereignissen vorgenommen. Die Auswirkungen auf die Umwelt werden nach den praktischen Ergebnissen der beschriebenen Hochwasserereignisse bewertet. Aus den physikalischen und chemischen Eigenschaften von Heizöl, insbesondere unter den spezifischen Bedingungen von Hochwasserereignissen, wird ein prinzipielles Szenarium bei Freisetzung von Heizöl nach Hochwasserereignissen entwickelt. Dazu werden für die Verteilung und den Abbau von Heizöl in der Umwelt Modellrechnungen einbezogen und mit experimentellen sowie praktischen Ergebnissen verglichen. Am konkreten Beispiel der Freisetzung von Heizöl beim Hochwasserereignis in Dessau-Waldersee im August 2002 wird die Heizölfreisetzung quantitativ bewertet.

Aus den erarbeiteten Ergebnissen sind in Deininger et al. (2006a) Konsequenzen und praktische Maßnahmen sowohl für die entsprechenden Körperschaften und Behörden als auch für Betroffene abgeleitet und bewertet.

\section{Heizölfreisetzung nach Hochwasserereignissen}

\subsection{Oder-Hochwasser 1997}

Von den überschwemmten Flächen wurden vom brandenburgischen Landesamt für Umwelt Schlamm- und Bodenproben entnommen und auf ihre Gehalte an umweltrelevanten Schadstoffen untersucht. Nur an Stellen, an denen Heizöltanks ausgelaufen waren, wurden erhöhte Gehalte an Mineralkohlenwasserstoffen festgestellt. Die Werte lagen aber alle nach Angaben des Landesamtes unter den für Brandenburg geltenden Grenzwerten für Böden in Wasserschutzgebieten.

\subsection{Donau-Hochwasser 1999}

Die Messungen an den ölverunreinigten Standorten zeigten erwartungsgemäß höhere Belastungen, die vereinzelt bis in Bodentiefen von 60 bis $90 \mathrm{~cm}$ - wenn auch dort in sehr niedrigen Konzentrationen - nachweisbar waren. Wiederholungsmessungen nach zwei Monaten zeigten, dass die Belastungen durch Mineralöl an allen untersuchten Standorten bereits um mehr als die Hälfte und bei über $50 \%$ der Standorte bereits auf unter $10 \%$ der anfänglichen Werte zurückgegangen waren. Das natürliche Abbauvermögen des Bodens bei Mineralölverunreinigungen ist somit sehr hoch. Es hat sich auch gezeigt, dass durch einfache Maßnahmen, wie z. B. Umpflügen, der Abbau noch wesentlich beschleunigt werden kann. Die Untersuchungen wurden noch bis zum Frühjahr 2000 fortgesetzt. Nachhaltige Beeinträchtigungen des Bodens durch andere Schadstoffe wurden bei den Messungen nicht festgestellt.

Hinsichtlich der Heizölgeruchsbelästigungen hat das Bayerische Landesamt für Umweltschutz wiederholt Messungen der Außenluft sowie in Innenräumen von verschie- 
denen Anwesen in Überschwemmungsgebieten durchgeführt. Die Ergebnisse der Außenluftmessungen zeigten, dass eine Gesundheitsgefährdung ausgeschlossen werden kann. In stark vom Hochwasser beschädigten Anwesen war bei späteren Messungen in einzelnen Räumen noch ein deutlicher Heizölgeruch wahrnehmbar, der jedoch wesentlich geringer war als unmittelbar nach dem Hochwasser. Die in einigen Innenräumen gemessenen Benzolkonzentrationen von $7-8 \mu \mathrm{g} / \mathrm{m}^{3}$ sind im Vergleich zur Hintergrundbelastung in ländlichen Gebieten erhöht, liegen aber in einem für verkehrsbelastete Stadtgebiete typischen Bereich.

\subsection{Donau-Hochwasser in Österreich im August 2002}

In den überfluteten Gemeinden wurde das ausgelaufene Heizöl durch spezialisierte Firmen umwelt- und fachgerecht entsorgt. Durch rechtzeitiges Absaugen im Kellerbereich wurden ein Eintrag in Oberflächengewässer, ein Versickern in den Boden sowie ins Grundwasser und somit ein großer Umweltschaden verhindert. Bleibende Schäden waren nicht nachweisbar.

\subsection{Elbe-Hochwasser in Sachsen im August 2002}

Im Landkreis Riesa-Großenhain sind beachtliche Heizölmengen freigesetzt worden. Freigesetzt wurden 247.1901 Heizöl, die abgesaugte Öl-Wasser-Menge betrug 492.5281, und 272 Grundstücke waren mit Heizöl kontaminiert. Mehrere Wohngebäude wurden durch ausgetretenes Heizöl stark belastet, die vollständig saniert bzw. abgerissen werden mussten. Röderau-Süd wurde nachträglich zum Überschwemmungsgebiet erklärt und abgerissen. In der übrigen Umwelt wie Boden, Oberflächengewässer und Grundwasser konnten keine Schäden nachgewiesen werden.

3.5 Elbe- und Mulde-Hochwasser in Sachsen-Anhalt im August 2002 (Autorenkollektiv 2003)

An 49 Standorten wurden Schlickproben von Kindergärten, Spielplätzen, Schulgärten, Sportplätzen und Gartenanlagen auf Mineralölkohlenwasserstoffe untersucht. Die Konzentrationen an Mineralölkohlenwasserstoffen in den Bodenproben der Spielplätze, Kindergärten, Schulgärten und Sportplätze lagen bis auf eine Ausnahme $(710 \mathrm{mg} / \mathrm{kg}$ TS) alle unterhalb des Eingriffswertes für sensible Nutzung entsprechend der Brandenburger Liste von $500 \mathrm{mg} / \mathrm{kg}$ TS.

Erhöhte Werte traten nur bei Schlickproben in Gartenanlagen auf. Es wurde frisch abgelagerter Schlick in einer Entnahmetiefe von $0-5 \mathrm{~cm}$ beprobt sowie zur Kontrolle bei einem Garten eine Probe unterhalb des Schlicks in einer Tiefe von $5-25 \mathrm{~cm}$ entnommen. Bei den Proben mit der Beprobungstiefe von 0-5 cm wurden Konzentrationen zwischen $5300-12.500 \mathrm{mg} / \mathrm{kg}$ TS ermittelt. Der Mineral- ölkohlenwasserstoffgehalt der Probe, die an der Stelle mit der höchsten Konzentration im oberflächennahen Bereich $(12.500 \mathrm{mg} / \mathrm{kg}$ TS; Beprobungstiefe $0-5 \mathrm{~cm})$ entnommen wurde, lag bei einer Tiefe von $5-25 \mathrm{~cm}$ bei $2900 \mathrm{mg} / \mathrm{kg}$ TS . Es ist also eine deutliche Abnahme des Gehaltes an Mineralölkohlenwasserstoffen ab einer Tiefe von $5 \mathrm{~cm}$ festzustellen. Bleibende Schäden waren nicht nachweisbar.

3.6 Hochwassersituation in Dessau-Waldersee (Elbe-Mulde-Winkel) (Helbig 2003; Schneider 2003)

Das Hochwasser der Mulde sowie der Elbe wurde durch das Landesamt für Gesundheits-, Umwelt- und Verbraucherschutz Sachsen-Anhalt zweimal täglich auf sämtliche Schadstoffe untersucht. Die Ergebnisse belegen, dass zu keinem Zeitpunkt besorgniserregende Schadstofffrachten $\mathrm{zu}$ verzeichnen waren.

Auch die Untersuchungsergebnisse des Ortsteiles von Waldersee, deren Beprobung durch das Amt für Umweltund Naturschutz in Auftrag gegeben wurde, erbrachten die gleiche Aussage, sofern nicht standortbezogene lokale Mineralölkohlenwasserstoffe (MKW) durch ausgelaufenes Heizöl nachgewiesen wurden.

Im Herbst 2002 wurden umfangreiche Beprobungen in Vor- und Hausgärten sowie in 17 Kleingärten durch das Amt für Umwelt- und Naturschutz in Auftrag gegeben. Hauptschwerpunkt waren Untersuchungen hinsichtlich der MKW-Belastungen, wobei auch in einigen Fällen weitere Schadstoffe, wie Schwermetalle und $\mathrm{B}-\mathrm{HCH}$, in die Untersuchungen einbezogen wurden. Hier belegten die analytischen Ergebnisse der Bodenproben nur geringfügige Belastungen mit MKW. Die anderen Schadstoffe konnten entweder nicht nachgewiesen werden oder lagen weit unter den Grenzwerten.

Das allgemein beobachtete Absterben von Pflanzenteilen oder ganzen Pflanzen auch in den Herbst hinein ist auf den aufgetretenen Sauerstoffmangel zurückzuführen. Bestimmte Pflanzenarten reagierten auch sehr empfindlich auf Staunässe, die in vielen Gärten aufgrund der Bodenbeschaffenheit und des lange anstehenden Grundwassers vorhanden war.

Eine weitere Bodenbeprobung wurde im Frühjahr 2003 durch das Amt für Umwelt- und Naturschutz in Auftrag gegeben. Hier wurden nochmals Bodenproben aus verschiedenen Hausgärten und Kleingartenanlagen entnommen. Die Bodenproben wurden wiederum auf Rückstände von Heizöl (MKW) untersucht. In den Fällen, in denen im Herbst noch deutliche Spuren von MKW nachgewiesen wurden, zeigten die Ergebnisse, dass ein weitgehender mikrobiologischer Abbau erfolgt war. Nach Auswertung aller im Amt für Umwelt- und Naturschutz vorliegenden Untersuchungsergebnisse mit den Analysen des Bodens aus der letzten Beprobung (März-Mai 2003) der einzelnen Grundstücke und Gärten kann eingeschätzt werden, dass keine schädlichen 
Bodenveränderungen, die auf das Hochwasser im August 2002 zurückzuführen sind, vorliegen.

\subsection{Mulde-Hochwasser im Raum Bitterfeld (Brack et al 2002)}

Als Belastungsquellen werden u. a. auch leckgeschlagene Öl- und Benzintanks im Oberlauf der Mulde angegeben. Insbesondere in Gebieten, wo die Überschwemmungswässer durch geplatzte Öltanks mit Mineralöl verunreinigt wurden, können toxische Inhaltstoffe in erhöhtem Maße transportiert worden sein. Inwieweit hier tatsächlich auch ein Eintrag in den Oberboden stattgefunden hat, wäre mit stichprobenartigen Analysen zu klären. Die durch Öl kontaminierten Flächen in Gartenanlagen von Bitterfeld, Jeßnitz und Raguhn wurden bis zu einer Tiefe von ca. $5 \mathrm{~cm}$ abgetragen und entsorgt. Bleibende Schäden waren nicht nachweisbar.

\subsection{Elbe-Hochwasser östlich von Magdeburg}

(Autorenkollektiv 2003)

Obwohl es im privaten Bereich zur Freisetzung von Heizöl gekommen ist, spielt die Belastung mit Mineralölkomponenten in der Umwelt, von wenigen, lokal begrenzten Ausnahmen abgesehen, keine Rolle.

\section{Umweltverhalten von HeizöI}

Zusammensetzung Heizöl (Sorte EL) besteht aus einem komplexen Gemisch von Kohlenwasserstoffen mit einem Siedebereich von 160 bis $390^{\circ} \mathrm{C}$ und einem Flammpunkt von $>55^{\circ} \mathrm{C}$. Die Kohlenwasserstoffe sind paraffinischer, naphthenischer (Cycloalkane) und aromatischer Natur (Alkylaromaten). Ihre Kohlenstoffzahl liegt zwischen $\mathrm{C}_{9}$ und $\mathrm{C}_{20}$.

Freisetzung in die Umwelt Von Unfällen bei Lagerung, Umfüllen und Transport abgesehen, kann es zur Heizölfreisetzung aus dem privaten Bereich bei Hochwasser kommen, wie sich im Sommer 2002 bei der Flut in Sachsen und Sachsen-Anhalt gezeigt hat. Allerdings täuschen die großen Öllachen auf dem Wasser über die tatsächlich freigesetzten Mengen, da diese Ölfilme oft nur äußerst geringe Schichtdicken aufweisen: So bedecken z. B. 1001 Öl eine Wasserfläche von $1 \mathrm{~km}^{2}$ mit einer Schichtdicke von nur $0,0001 \mathrm{~mm}$.

Heizöle werden auch bestimmungsgemäß in die Umwelt eingetragen, da sie direkt als Herbizide dienen oder diesen als Lösungsmittel zugesetzt werden. Noch immer betragen die angewandten Mengen 20-401 Heizöl pro Hektar. Neben Heizöl sind auch andere Kohlenwasserstofflösungsmittel ähnlicher Zusammensetzung im Pflanzenschutz im Gebrauch (Kurth 1968; Malkomes 2005).

Als dritte - ebenfalls nicht zu unterschätzende Eintragsquelle für Kohlenwasserstoffe - sind die Wasch- und Reini- gungsmittel und die Textilhilfsmittel zu nennen, wobei hier die Tendenz des Eintrages rückläufig ist.

Problem der Bewertung von Kontaminationen Kohlenwasserstoffe sind in der Natur ubiquitär verbreitet, sie werden ständig gebildet und unterliegen als Naturstoffe einem fortlaufenden Abbau. Hauptproduzenten von Kohlenwasserstoffen sind Pflanzen, (Meeres-)Mikroorganismen und in weit geringerem Ausmaß auch höhere Tiere. So liegen die normalen Gehalte der Böden bei ca. $100 \mathrm{mg}$ KW pro Kilogramm Boden oder höher, sodass als Prüfwerte $1000 \mathrm{mg} / \mathrm{kg}$ vorgesehen sind und die Maßnahmewerte für die Sanierung bei $5000 \mathrm{mg} / \mathrm{kg}$ Boden liegen (Sächsisches Landesamt für Umwelt und Geologie 2003; BBodSchV 1999).

Ein einfaches Nachweiskriterium (für eine Ölkontamination) besteht darin, zwischen den geradzahligen und den ungeradzahligen Kohlenwasserstoffen zu unterscheiden: In nichtkontaminierten Böden ist ein Übergewicht an ungeradzahligen Kohlenwasserstoffen zu erwarten, die auf pflanzlichen Ursprung zurückzuführen sind (Isoprenhypothese). Dagegen liegt bei fossilen Kohlenwasserstoffen ein ausgeglichenes Verhältnis vor, das auf mikrobiellen Ursprung, verbunden mit Folgeumwandlungen, hinweist. Durch metabolischen Umbau geht dieses Unterscheidungsmerkmal nach zwei bis drei Wochen verloren.

Verbleib des Heizöls nach seiner Freisetzung Für die Eliminierung des Heizöls in die Umwelt kommen drei Wege, die miteinander verbunden sind, in Betracht: Verdunstung, Fotooxidation und mikrobieller Abbau (Landesamt für Wasserwirtschaft 1986).

Für alle drei Eliminierungswege gilt, dass Heizöl keine diskrete Chemikalie ist, deren Verhalten man pauschal abschätzen kann, sondern dass es ein Kohlenwasserstoffgemisch ist, dessen Einzelkomponenten ein unterschiedliches Verhalten zeigen. Das Umweltverhalten von Heizöl hängt vom Verhalten der Einzelkomponenten ab. Um eine Bewertung dieses Verhaltens vornehmen zu können, wurden aus den genannten Stoffgruppen (Alkane, Cycloalkane, Aromaten) die Vertreter ausgewählt, deren Verhalten in der Umwelt untersucht worden ist und die daher als repräsentativ für ihre Stoffgruppe gelten können.

Verdunstung und Fotooxidation Unmittelbar nach der Heizölfreisetzung setzt die Verdunstung ein. Auf diese Weise wird die Menge an freigesetztem Heizöl sehr rasch um $25-40 \%$ reduziert. Es verdunsten die Heizölkomponenten mit Siedepunkten bis zu $299^{\circ} \mathrm{C}$, das ist der Bereich bis etwa $n$-C14, der vor allem die aromatischen Kohlenwasserstoffe und die Cycloalkane umfasst. Die Verdunstung hängt neben den inhärenten Stoffeigenschaften vor allem von der AuBentemperatur ab. Der Verdunstungsprozess ist meist nach wenigen Stunden, maximal nach $100 \mathrm{~h}$ (bezogen auf Rohöl) abgeschlossen.

An die Verdunstung unmittelbar schließt sich die Fotooxidation in Luft an (Landesamt für Wasserwirtschaft 
1986; DGMK-Projekt-413 1989; BUA 1992). Durch Sonnenlicht und bereits in der Troposphäre vorhandene Radikale werden die Kohlenwasserstoffe in sauerstoffhaltige Verbindungen umgewandelt. Das wirksamste Radikal ist das OH-Radikal („Waschmittel der Atmosphäre“), daneben spielen Ozon, das NO-Radikal u. a. eine Rolle. Olefine und Aromaten werden verhältnismäßig schnell abgebaut, ihre Lebenszeit in der Atmosphäre beträgt nur wenige Stunden oder Tage - dagegen haben $n$-Alkane eine Lebenszeit von mehreren Tagen. Im ungünstigsten Fall (gemeint sind die Komponenten des Rohöls) sind die Kohlenwasserstoffe erst in 1000 Tagen total abgebaut. Die im Heizöl vorhandenen Komponenten haben Halbwertszeiten von wenigen Stunden bis zu einigen Tagen.

Durch Regen können die fotooxidativen Stoffe wieder auf die Erde (Boden/Wasser) gelangen. Aufgrund der Tatsache, dass in die Ursprungsmoleküle bereits Sauerstoff eingefügt worden ist, sind diese oxidierten Stoffe dem mikrobiellen Abbau ohne Verzögerung zugänglich.

Die Fotooxidation in der Luft kann zum totalen Abbau führen oder den Abbau der Kohlenwasserstoffe erleichtern. Die Fotooxidation läuft auch im Wasser ab. Sie ist hier an die Anwesenheit von Fotosensibilisatoren gebunden, die entweder vorhanden sind oder aus mehrkernigen Aromaten gebildet werden. Auch in diesem Fall wird Sauerstoff in die Kohlenwasserstoffmoleküle eingeführt, sodass der mikrobielle Abbau sich rascher vollziehen kann.

Mikrobieller Abbau Als Naturstoffe unterliegen Kohlenwasserstoffe einem ständigen mikrobiellen Abbau. Die kohlenwasserstoffabbauenden Mikroorganismen sind ubiquitär verbreitet. Sie gehören verschiedenen Spezies an. In ölkontaminierten Böden und Sedimenten vollzieht sich der Abbau schneller als in nichtkontaminierten. Der Abbau kann durch „vorgeschaltete“ fotooxidative Umwandlungen, die mit der Einführung von Sauerstoff in das jeweilige Molekül verbunden sind, beschleunigt ablaufen. Die Fotooxidation ist jedoch keine Voraussetzung für den Abbau, die Mikroorganismen sind selbst in der Lage, Sauerstoff in die Moleküle einzuführen.

Der Abbau verläuft überwiegend aerob und daher an die Anwesenheit von Sauerstoff gebunden. Der anaerobe Abbau im Wasser konnte nachgewiesen werden. Er vollzieht sich vor allem in den Lebensgemeinschaften von Biofilmen und in den Matten der Cyanobakterien. Dabei verlaufen mikrobielle Abbauvorgänge immer an den Phasengrenzen im vorliegenden Fall Wasser-Öl. Die Grenze zwischen aerobem und anaerobem Abbau im Wasser wird bei $8 \mu \mathrm{g}$ gelöstem Sauerstoff pro Gramm Wasser gezogen. Der aerobe Abbau verläuft ca. 300-mal schneller als der anaerobe Abbau. Die sowohl im Labor als auch bei Tankerunfällen festgestellte Reihenfolge im Abbau lautet: $n$-Alkane > Isoprenoide $>$ Naphthene (Cycloalkane) und $n$-Alkane $>$ Aromaten (Ausnahmen möglich) (DGMK-Projekt-413 1989;
Holwitt 1997; Musat 2005). Im Allgemeinen ist der Abbau im Wasser innerhalb von 100 Tagen abgeschlossen.

Auch von den Mikroorganismen des Bodens werden Kohlenwasserstoffe gut abgebaut (Fabig 1988). Dabei vollzieht sich der Abbau in der oberen Bodenschicht relativ zügig, mit einer deutlichen Steigerung in den Sommermonaten. In der unteren Bodenschicht zwischen 30 und $46 \mathrm{~cm}$ vollzieht sich der Abbau langsamer und temperaturunabhängig. Mineralölkohlenwasserstoffe können nur im lockeren und humusarmen Boden (Sand) bis zu wasserführenden Schichten vordringen. Ein biologischer Abbau von Kohlenwasserstoffen im Grundwasser ist möglich, wenn Sauerstoff im Grundwasser vorhanden ist.

Einfluss auf die Organismen der Umwelt Zum Verhalten von Mineralölkohlenwasserstoffen in der Umwelt, insbesondere im Wasser und im Boden, liegen zahlreiche Untersuchungen vor, die vor allem über Verdunstung, Fotooxidation und mikrobiellen Abbau sowie über die Verteilung im Boden Auskunft geben. Dazu kommt eine Vielzahl von experimentellen Arbeiten, die Aufschluss über detaillierte Abbaumechanismen geben. Dagegen ist der Einfluss der Kohlenwasserstoffe auf die im Wasser und im Boden lebenden Organismen nur sporadisch untersucht (BUA 2000; BUA 1992a,b; Deininger et al. 2006b; Hock und Elstner 1988; Malkomes 2005).

Unmittelbar nach der Ölfreisetzung kann es zu Schäden an Tieren, Pflanzen und Mikroorganismen kommen. Diese sind vor allem auf die physikalischen Eigenschaften der Öle zurückzuführen, durch die es zu Verklebungen des Gefieders von Vögeln, zu Verklebungen der Kiemen von Fischen und zu Verklebungen der Spaltöffnungen von höheren Pflanzen kommt. Ferner führen die Öle in hohen Konzentrationen zur Auflösung der Membranen der Zellen von höheren Pflanzen und nicht zuletzt wird auch das Trinkwasser ungenießbar (Oehlmann und Markert 1999). Das Absterben von Wasserorganismen geht weniger auf die Freisetzung von Heizöl zurück als auf den Sauerstoffmangel durch den an der Wasseroberfläche herabgesetzten Luftaustausch und die damit verbundene geringere Sauerstoffaufnahme des Wassers, dazu kommt der hohe Sauerstoffverbrauch durch die absterbenden Pflanzen und die zugleich freigesetzten Fäkalien (insbesondere Gülle). Als ungünstig erwies sich auch die Wärme (August). Alle diese Faktoren trugen dazu bei, dass der Sauerstoffmangel der Fließgewässer sich dem kritischen Wert von $4 \mathrm{mg} / \mathrm{l}$ näherte, der aber noch immer weit über der Grenze des anaeroben Ölabbaus liegt.

Akute Schäden durch Heizöl an der Mikroflora und Fauna des Bodens sowie des Wassers sind nicht von vornherein auszuschließen. So liegen die Schwellenwerte der Schadwirkung für einige Wasserorganismen unterhalb von $1 \mathrm{mg} / \mathrm{l}$. Als besonders empfindlich erweisen sich Fischnährtiere und einige Fischarten. Bei letzteren wird durch Kohlenwasser- 
stoffe die Reproduktion bereits bei Konzentrationen $<1 \mathrm{mg} / 1$ beeinträchtigt.

Diese Konzentrationen werden bei Freisetzung von Heizöl nach Hochwasserereignissen meist weit unterschritten. So zeigten die Messungen des Sondermessprogramms zum Hochwasser im August 2002 in Sachsen-Anhalt Werte für den Kohlenwasserstoffindex (KWI) $\leq 0,1 \mathrm{mg} / \mathrm{l}$ (Autorenkollektiv 2003).

\section{Praxisbezogene Bewertungen von Verteilung und Abbau von Heizöl in der Umwelt bei Freisetzung nach Hochwasserereignissen}

\subsection{Prinzipielles Szenarium (Deininger et al 2006a)}

Die Verteilung und Umwandlung von Heizöl sowie die Beseitigung von Ölfilmen bei Freisetzung nach Hochwasserereignissen sind schematisch in Abb. 1 veranschaulicht. Das nach dem Austritt auf dem Wasser schwimmende Öl soll durch Barrieren eingegrenzt und soweit als möglich abgepumpt und entsorgt werden, um großflächige Ausbreitungen und Beeinträchtigungen von Wasseranlagen sowie Schäden an Häusern weitgehend zu verhindern. Trotzdem kann in Wohngebieten ein Teil des Öls in das umgebende Mauerwerk (Wände, Decke) des Gebäudes bei beschädigten Heizölanlagen eintreten, ein anderer Teil verteilt sich auf die umgebende Wasserfläche bzw. wird über die Kanalisation abfließen.

Die Analyse der Daten für ein konkretes Ereignis der Heizölfreisetzung beim Elbe-Hochwasser 2002 nach einem
Deichbruch in Dessau-Waldersee ergab folgende Verteilung: Aus 190 betroffenen privaten Ölheizungen wurden $540 \mathrm{~m}^{3}$ Heizöl freigesetzt, von dem ca. $40 \%$ abgesaugt und separiert werden konnten, $4 \%$ wurden durch die Kanalisation abgeführt, $3 \%$ trat in das Mauerwerk von Gebäuden ein, ca. $40 \%$ der ausgetretenen Ölmenge verdunstete innerhalb von zwei Tagen, und 3\% traten nach dem Abtrocknen mit einer Konzentration von ca. 300 mg Öl pro Kilogramm Boden in den Boden ein und wurden hier mikrobiell abgebaut (Schneider 2003).

\subsection{Ausbreitung, Verdunstung und Abbau von Heizöl auf Wasseroberflächen}

Das Verhalten von Öl auf Wasseroberflächen wird durch seine physikalischen und chemischen Stoffeigenschaften bestimmt (Oebius 2004). Mit dem Heizölaustritt laufen verschiedene Prozesse der Verteilung und der Umwandlung von Heizöl nebeneinander ab. Ausgetretenes Öl schwimmt wegen seiner geringen Dichte $\left(820-860 \mathrm{~kg} / \mathrm{m}^{3}\right)$ auf der Wasseroberfläche und kann bei entsprechender Strömung mit dem Wasser fortgetragen werden. In diesem Fall werden lokale Beeinträchtigungen durch Heizöl in der Umgebung des Heizölaustritts verringert oder ganz vermieden.

Auf einer ruhigen Wasseroberfläche, z. B. in einem Polder, wird sich das freigesetzte Öl aufgrund seiner Viskosität und Oberflächenspannung rasch zu dünnen Ölfilmen ausbreiten - im Grenzfall bis zu einer monomolekularen Schicht. So haben z.B. bunt schillernde Ölteppiche auf Wasseroberflächen nur eine Schichtdicke von ca. $0,3 \mu \mathrm{m}$, d. h. 0,3 Millionstel Meter - weniger als ein Hundertstel der

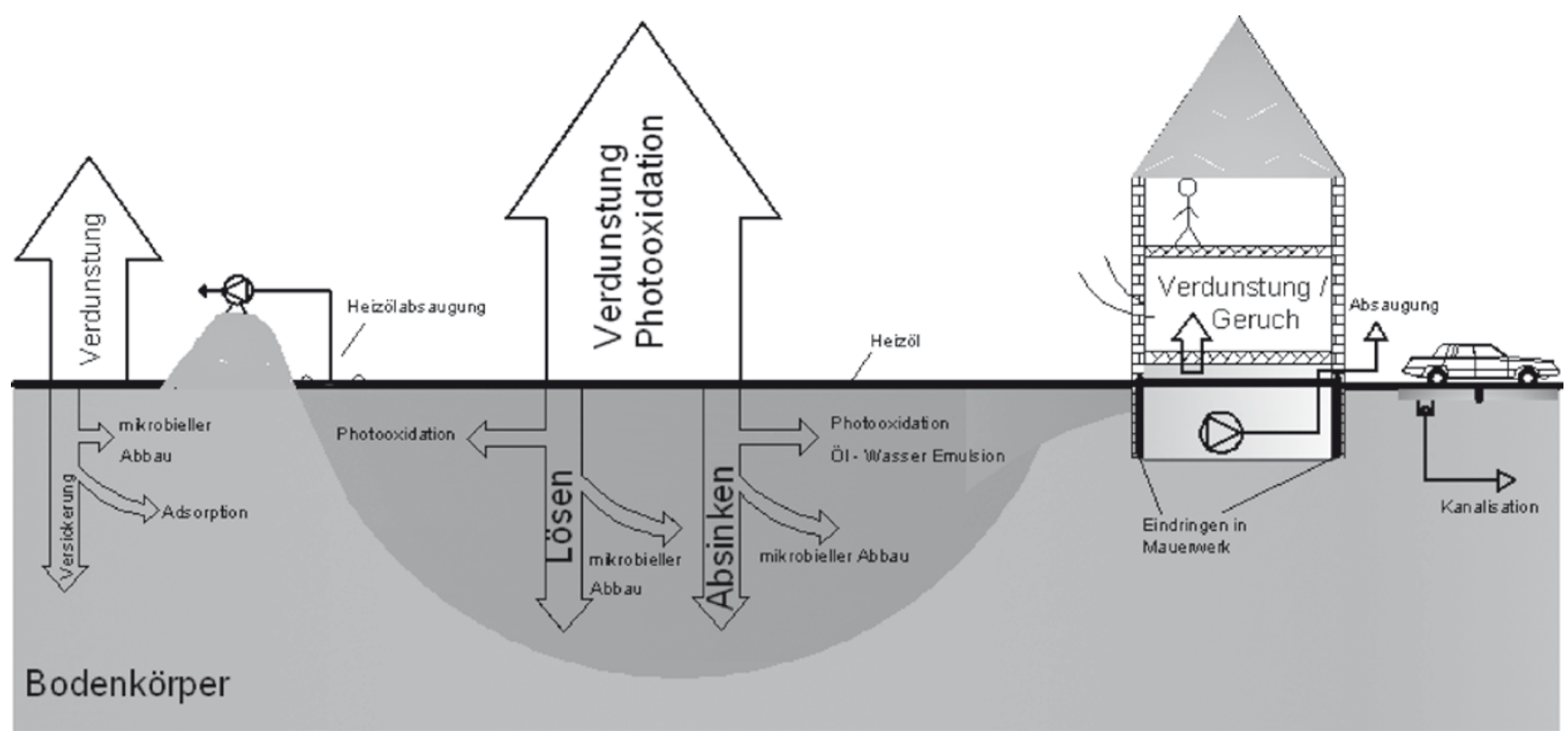

Abb. 1 Verteilung, Abbau und Beseitigung von Heizöl nach Freisetzung bei Hochwasserereignissen 
Tabelle 1 Ausbreitung und Verdunstung von $1 \mathrm{~m}^{3}$ Heizöl aus einer Lache bei $20^{\circ} \mathrm{C}$ und einer Windgeschwindigkeit von $2 \mathrm{~m} / \mathrm{s}$ (Deininger et al. 2006a). Ausbreitung nach Formel aus Oebius (2004), Verdunstung mit Programm 8FEUEX V 4.0

\begin{tabular}{cccc}
\hline Lachengröße von $\mathbf{1} \mathbf{~ m}^{\mathbf{3}}$ Heizöl & \multicolumn{2}{c}{ Verdunstung von $\mathbf{1} \mathbf{~ m}^{\mathbf{3}}$ Heizöl } \\
\hline Ausbreitungszeit & Oberfläche & Verdunstungs- & Verdunstungs- \\
& & strom & zeit \\
$(\mathrm{min})$ & $\mathrm{m}^{2}$ & $(\mathrm{~g} / \mathrm{s})$ & $(\mathrm{h})$ \\
1,7 & 500 & 10,9 & 21,68 \\
16,8 & 5000 & 96 & 2,46 \\
168,35 & 50.000 & 845,5 & 0,47 \\
\hline
\end{tabular}

Dicke eines Menschenhaares. Damit entspricht diese Öllache auf einer Fläche von $1 \mathrm{~km}^{2}$ nur einer Ölmenge von 3001 oder nur 31 Heizöl auf 1 ha Fläche.

Gleichzeitig mit der Ausbreitung auf der Wasseroberfläche tritt ein Teil des Öls durch Verdunstung in die Atmosphäre über. Dieser Anteil beträgt bei Heizöl 40-45\%. Die Verdunstung hängt von der chemischen Zusammensetzung, dem Dampfdruck sowie von äußeren Faktoren wie der Temperatur, der Windgeschwindigkeit, der Menge des Öls und der Dicke des Ölfilms ab. Bei Heizöl erfolgt in Sommermonaten die Verdunstung innerhalb von zwei bis drei Tagen.

In Tabelle 1 sind berechnete Werte für die Ausbreitungsgeschwindigkeit von Heizöl auf Wasseroberflächen und die Verdunstungsraten angegeben (Deininger et al. 2006a).

Mit dem Übergang in die Atmosphäre beginnt der Abbau der verdunsteten Heizölbestandteile durch Fotooxidation (BUA 1992). Die Fotooxidation der Kohlenwasserstoffe führt zu sauerstoffhaltigen Verbindungen, die an atmosphärischen Reaktionen teilnehmen und entweder in der Atmosphäre vollständig abgebaut werden oder durch trockene bzw. feuchte Deposition wieder auf die Erde gelangen und hier dem mikrobiellen Abbau unterliegen.

Die Heizölbestandteile, die nicht verdunsteten, sondern sich auf dem Wasser zu einem dünnen Ölfilm ausgebreitet haben, werden nur zu einem sehr geringen Teil im Wasser gelöst. Heizöl ist in Wasser kaum löslich $(\leq 5 \mathrm{ppm}$ bei $20^{\circ} \mathrm{C}$ nach Sicherheitsdatenblatt Heizöl Extra leicht), und auch die Menge des dispergierten Öls ist sehr gering (ca. $1,5 \mathrm{mg} / \mathrm{l}$ ) (io-warnemünde 2001).

Die gelösten Stoffe unterliegen dann im Wasser sowohl dem fotooxidativen als auch vorwiegend dem mikrobiellen Abbau. Der Beitrag der Fotooxidation im Wasser zur Ölentfernung aus der Umwelt wurde auf eine tägliche Menge von $0,1 \%$ des gesamten Öls beziffert. Bei zehn Ölen konnte mit $86-99 \%$ in 30 Tagen eine hohe Effektivität der Fotooxidation von Aromaten nachgewiesen werden (Brack et al. 2002). Die gelösten Bestandteile werden im Wasser je nach Jahreszeit und Klimabedingungen in ein bis zwei Monaten abgebaut.

Obwohl gelöste Bestandteile von Mineralölkohlenwasserstoffen auch toxische Eigenschaften haben und damit
Fische, Kleintiere und Mikroorganismen im Wasser bei entsprechenden Konzentrationen unmittelbar schädigen können (Hock und Elstner 1988), wurden bei bisherigen Hochwasserereignissen diese direkten Schädigungen nach Heizölaustritten nicht beobachtet. Anders ist die Situation bei Transport- und Abfüllunfällen auf Flüssen sowie in Hafenanlagen, bei denen größere Ölmengen in das Wasser gelangen!

Bei turbulenten Wasserströmungen können sich unter bestimmten Bedingungen relativ beständige Öl-in-Wasserund Wasser-in-Öl-Emulsionen bilden, die sowohl fotooxidativ als auch mikrobiell langsamer abgebaut werden und/ oder sich im Sediment ablagern können. Auch hierdurch ist keine direkte Schädigung der aquatischen Flora und Fauna bei bisherigen Heizölaustritten nach Hochwasser beschrieben worden.

\subsection{Ausbreitung und Abbau von Heizöl im Boden}

Nach Abfließen bzw. Abtrocknen des stehenden Wassers verbleiben die restlichen Ölbestandteile im Schlamm bzw. dringen in den Bodenkörper ein. Die Verteilung des Öls und insbesondere auch die Eindringtiefe des Öls in der oberen ungesättigten Bodenschicht hängen von verschiedenen Parametern wie Bodenart und -struktur, vom Wassergehalt des Bodens sowie von physikalisch-chemischen Parametern ab (Fabig 1988; Hettwer et al. 2004). Somit ist die Aussage über Eindringtiefe und Eindringgeschwindigkeit vom konkreten Ort abhängig.

Für die Betrachtung des Eindringens in das Grundwasser durch Heizöleintritte in den Boden sind die Penetration des Öls im Boden, das Speichervermögen und das große Selbstreinigungspotenzial des Bodens von Bedeutung.

Für das Versickerungs- und Wanderungsverhalten von KW ist die Bodenstruktur eine bestimmende Größe. Bei stark durchlässigen Böden wie Kies- oder groben Sandboden mit einem großen Anteil an Grobporen versickern KW sehr schnell. Bei feinkörnigen, trockenen Sandböden nimmt die KW-Fraktion hauptsächlich die kleineren Poren ein, während die größeren Poren im Wesentlichen mit Luft gefüllt bleiben.

Voraussetzung für die vertikale Bewegung von Mineralölprodukten ist, dass eine solche Menge Mineralöl vorliegt (und nachströmen kann), die über der Sättigung liegt. Die Kohlenwasserstoffe benetzen auf ihrem Weg durch den Boden die passierten Bodenpartikel, und ein Teil der Kohlenwasserstoffe wird adsorbiert. Wenn die Menge an nachströmenden Kohlenwasserstoffen begrenzt ist, kann es dazu kommen, dass sämtliche in den Boden eingedrungenen Kohlenwasserstoffe an den Bodenpartikeln adsorbiert sind (= immobile oder restliche Sättigung). Ist dieser Zustand erreicht, erlischt die vertikale und horizontale Bewegung der Kohlenwasserstoffe. In Tabelle 2 sind Anhaltswerte für die 
Tabelle 2 Anhaltswerte der Residualsättigung (in $\mathrm{mg} / \mathrm{kg}$ ) für verschiedene Lockergesteine (entnommen aus Handbuch zur Altlastenbehandlung 2006)

\begin{tabular}{lrrcr}
\hline & Benzin & Diesel & Motorenöl $^{\mathbf{a}}$ & Schweröl \\
\hline Grobkies & 200 & 950 & 3200 & 4800 \\
Feinkies & 2000 & 4500 & 7000 & 12.000 \\
Grobsand & 2800 & 5600 & 9000 & 15.000 \\
Mittelsand & 4500 & 9500 & 17.000 & 25.000 \\
Feinsand/Schluff & 7500 & 17.000 & 26.000 & 39.000 \\
\hline
\end{tabular}

${ }^{a}$ Mittlere kinematische Viskosität $18 \mathrm{~mm}^{2} / \mathrm{s}$

Restsättigung in verschiedenen Lockergesteinen aufgeführt (Handbuch zur Altlastenbehandlung 2006).

Kommt es zu Regenfällen, dann kann das Wasser bereits sorbierte Kohlenwasserstoffe wieder desorbieren und in tiefere Zonen des Bodens verschieben. Allerdings geht die Verdrängung der Kohlenwasserstoffe sehr langsam vor sich: um 1 Teil Kohlenwasserstoffe zu eluieren, sind 40.000 Teile Wasser erforderlich. Je nach Porosität des Bodens kommt es beim Eindringen von Kohlenwasserstoffen zu typischen Ausbreitungsmustern: Bei einem durchlässigen, grobporigen und trockenen Boden breiten sich die Kohlenwasserstoffe in Form schlanker Keulen aus, ist die Durchlässigkeit geringer, stumpft die Keule ab, die Eindringtiefe wird geringer, bei verschieden durchlässigen Schichten (z. B. bei einem typischen Podsol) entwickeln sich Ausbreitungsmuster mit alternierenden Formen.

Neben der Porosität und der chemisch bedingten Struktur des mineralischen Anteils des Bodens sind die organischen Bestandteile des Bodens entscheidend für Wanderungsverhalten, Eindringtiefe und schließlich für den Abbau der Kohlenwasserstoffe. Die organischen Bestandteile können 3 bis $7 \%$ des Gesamtbodens ausmachen. Von dieser organischen Bodenfraktion bilden 5\% die Bodenflora und -fauna, 10\% tote sowie belebte Wurzelmasse und ca. 85\% Humus, die postmortalen organischen Substanzen, die einem ständigen Ab-, Um- und Aufbau unterliegen. Die Huminstoffe sind reich an hydrophoben Bindungsstellen und haben dadurch eine hohe Sorptionskapazität für hydrophobe Moleküle wie Kohlenwasserstoffe. Daher ist die Wanderungstendenz der $\mathrm{KW}$ in humushaltigen, vor allem in humusreichen Böden stark herabgesetzt. Die Bindung von Kohlenwasserstoffen an Huminstoffe kann so stark sein, dass die Kohlenwasserstoffe quasi an den Huminstoffen fixiert sind und ihre chemischen Eigenschaften völlig verlieren. Auch ihr analytischer Nachweis kann durch eine sehr starke Bindung total maskiert werden.

Andererseits können Huminstoffe auch als Emulgatoren fungieren, so setzten z.B. Na-Salze von Huminstoffen die Oberflächenspannung von Wasser herab. Damit können unlösliche organische Verbindungen in Lösung gebracht wer-

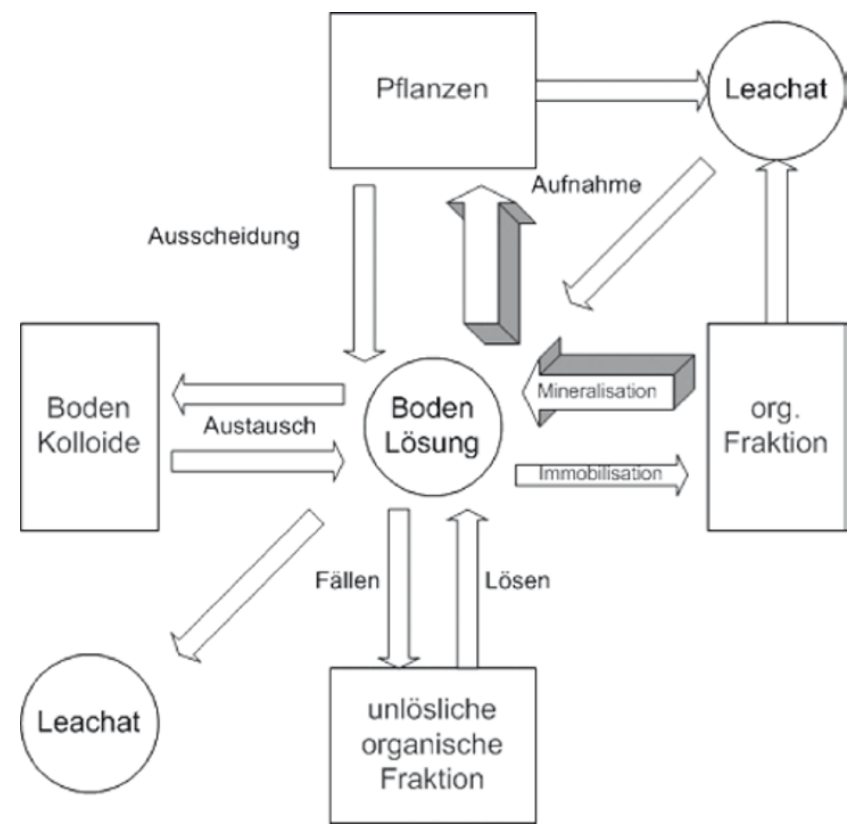

Abb. 2 Zentrale Stellung der Bodenlösung für Verteilungs- und Umsetzungsprozesse in einem Boden (Fabig 1988)

den. Wenn Kohlenwasserstoffe in den Boden eindringen, bildet sich ein Vier- bzw. Fünfphasensystem:

1. feste Phase: mineralische und organische Bodenbestandteile,

2. flüssige Phase: Wasser/Kohlenwasserstoffe,

3. gasförmige Phase: Luft und

4. Phase: belebte, organische Biomasse.

Die Bestandteile dieses Systems treten miteinander in Wechselwirkung (Abb. 2).

Im Allgemeinen werden Ölkontaminationen aus Hochwasserereignissen in Böden nicht bis in das Grundwasser vordringen und Wasser führende Schichten nicht oder nur in geringem Maße erreichen. Die Hauptmenge verbleibt in den oberen Bodenschichten. So konnte experimentell belegt werden, dass selbst 121 Rohöl pro $\mathrm{m}^{2}$, aufgebracht auf einen organisch reichen Boden, nach einem Jahr nur $4 \mathrm{~cm}$ in den Boden eingedrungen waren (Fabig 1988).

Nach dem Eindringen von Heizöl in den Boden findet die Abnahme der eingetragenen Kohlenwasserstoffe durch Austrag mit der Bodenluft und vor allem durch mikrobiellen Abbau statt. An den meisten Böden existiert eine den Standortbedingungen angepasste natürliche Mikroflora, die in der Lage ist, Mineralölkomponenten abzubauen. Unter den klimatischen Bedingungen in Deutschland ist der Abbau von Mineralölkohlenwasserstoffen bei Temperaturen zwischen 5 und $30^{\circ} \mathrm{C}$ gewährleistet. Dabei wirken sich klimatische und jahreszeitliche Temperaturschwankungen auf die Abbauraten aus. Die größte mikrobielle Aktivität in den Böden liegt bei einem Wassergehalt von 
etwa $50 \%$ der maximalen Wasserhaltekapazität des Bodens, wie sie in Deutschland im Jahresmittel auch an vielen Orten erreicht wird. Danach betragen in Abhängigkeit von Bodenart und klimatischen Bedingungen die Halbwertszeiten für den natürlichen Ölabbau in Böden zwei bis vier Monate, d.h. in diesen Zeiten wird die Ölkonzentration im Boden auf die Hälfte abgebaut. Abbauraten für Dieselölkontaminationen in Ackerböden von $70 \%$ in drei Monaten werden beschrieben (Hettwer et al. 2004; Riis et al. 1998).

Die Auswertung der beschriebenen Hochwasserereignisse zeigte auch, dass durch Heizölaustritte bisher keine Grundwasserleiter betroffen waren. Die Messungen der untersuchten Hochwasserereignisse ergaben Gehalte an Kohlenwasserstoffen in Böden, die i. Allg. weit unterhalb des Eingriffswertes für sensible Nutzung $(500 \mathrm{mg} / \mathrm{kg}$ Boden entsprechend der Brandenburger Liste) liegen, wobei eine deutliche Abnahme des Gehaltes an Kohlenwasserstoffen ab einer Tiefe von $5 \mathrm{~cm}$ festzustellen ist (Autorenkollektiv 2003).

Selbst bei der Sanierung von Mineralölschäden nach Transportunfällen bzw. an Altstandorten mit wesentlich höheren Bodenkontaminationen an Mineralölkohlenwasserstoffen als bei Heizölfreisetzung nach Hochwasserereignissen werden durch gezielte on-site Behandlung der Böden mit ihren natürlichen Mikroorganismen hohe Abbauleistungen erreicht und in wenigen Monaten die Konzentrationen an Kohlenwasserstoffen um 70-90\% reduziert (Held et al. 1997).

\section{Schlussfolgerungen}

Die praktischen Erfahrungen zeigen, dass bei Heizölkontaminationen von Böden, insbesondere nach Hochwasserereignissen, in Abhängigkeit von der eingedrungenen Ölmenge, der Bodenart und den klimatischen Bedingungen in Europa Kohlenwasserstoffe innerhalb weniger Wochen und Monate abgebaut sind. Diese Aussage bezieht sich insbesondere auf Heizöl und ist auf die relativ leicht abbaubaren Kohlenwasserstoffe des Heizöls und auch auf die universelle Verbreitung ölabbauender Mikroorganismen im Boden zurückzuführen.

Im Allgemeinen sind daher bei Heizölaustritten nach Hochwasserereignissen auf Bodenflächen keine Bodenaustauschmaßnahmen erforderlich. Ausreichend ist die Unterstützung des großen Selbstreinigungspotenzials des Bodens durch Belüften und erforderlichenfalls entsprechende Nährstoffzufuhr. Diese Maßnahmen reichen aus, um die eingetragenen Kohlenwasserstoffe des Heizöls in wenigen Monaten abzubauen (Sächsisches Landesamt für Umwelt und Geologie 2003; Fabig 1988; Hettwer et al. 2004). In bestimmten Situationen, beim Austritt größerer Heizölmengen in biologisch sensiblen Bereichen, in Wasserschutzgebieten und insbesondere in Wohngebieten, sollten aktive Maßnahmen wie der Einsatz von Ölsperren und Absaugen eingesetzt werden, um großflächige Ausbreitungen und Folgeschäden zu verhindern.

\section{Literatur}

Autorenkollektiv (2003) Das Hochwasser von Elbe und Mulde im August 2002 im Land Sachsen-Anhalt. Landesamt für Umweltschutz (LAU) und Landesanstalt für Landwirtschaft und Gartenbau (LLG), Halle

BBodSchV (1999) Bundesbodenschutz und Altlastenverordnung vom 12. Juli

Brack W, Altenburger R, Dorusch F, Hubert A, Möder M, Morgenstern P, Moschütz S, Mothes S, Schirmer K, Wennrich R, Wenzel K-D, Schüürmann G (2002) Hochwasser 2002. Umweltwiss Schadst Forsch 14(4):213-220

BUA (1993) OH-Radikale in der Troposphäre; Konzentration und Auswirkungen, Bd. 100. Hirzel S (Hrsg) BUA-Stoffberichte. Wissenschaftliche Verlagsgesellschaft, Stuttgart

BUA (1993a) Tetrahydronaphthalin, Bd. 101. Hirzel S (Hrsg) BUAStoffberichte. Wissenschaftliche Verlagsgesellschaft, Stuttgart

BUA (1993b) Decahydronaphthalin, Bd. 218. Hirzel S (Hrsg) BUAStoffberichte. Wissenschaftliche Verlagsgesellschaft, Stuttgart

BUA (1997) Benzin (Ottokraftstoffe). Hirzel S (Hrsg) BUA-Stoffberichte. Wissenschaftliche Verlagsgesellschaft, Stuttgart

Deininger D, Kardos J, Stephan U, Strobel U (2006a) Studie Schäden durch Heizöl nach Hochwasserereignissen. Studie im Auftrag von IWO Institut für wirtschaftliche Oelheizung e. V., Hamburg

Deininger D, Kardos J, Stephan U, Strobel U (2006b) Studie Schäden durch Heizöl nach Hochwasserereignissen. Studie im Auftrag von IWO Institut für wirtschaftliche Oelheizung e. V., Hamburg, Anlagen 9, 10

DGMK-Projekt-413 (1989) Untersuchungen zum mikrobiellen Abbau von Mitteldestillaten im Meer. DGMK 2005, Hamburg

Fabig W (1988) Mikrobiologie und Chemie bei der Verunreinigung von Boden und Grundwasser. In: Umwelttechnik, Bd. 164. Schweinsfurth R (Hrsg) Angewandte Mikrobiologie der Kohlenwasserstoffe in Industrie und Umwelt. expert-Verlag, Renningen

Handbuch zur Altlastenbehandlung (2006) Freistaat Sachsen, Landesamt für Umwelt und Geologie, Dresden, Teil 7: Anlagen

Helbig B (2003) Die Hochwasserkatastrophe im August 2002 in Dessau, 2. Aufl. Rupa-Druck, Dessau

Held Th, Stempel R, Bohlen D (1997) Handbuch der Altlasten, 2. Aufl. EF-Verlag, Neuruppin

Hettwer K, Warrelmann J, Heyser W, Martus P,Gaab S, Püttmann W, Drewes U, Walter U, Vehlhaber D (2004) Langzeituntersuchungen zu den Möglichkeiten und Grenzen der Nutzung natürlicher Selbstreinigungsprozesse für ausgewählte Schadstoffe am Beispiel kontaminierter militärischer Liegenschaften, Literaturstudie. Bericht Oktober 2004, BMU Umweltforschungsplan 1998, Berlin Dessau

Hock B, Elstner EF (1988) Schadwirkungen auf Pflanzen. BJ Wissenschaftsverlag, Mannheim Wien Zürich

Holwitt U (1997) Erdölabbau am Persischen Golf nach der Ölpest von 1991. Dissertation Fachbereich Chemie der Math.-Nat. Fakultät der Westfälischen Wilhelmsuniversität, Münster

www.io-warnemuende.de/research/de_oel_allgemein (2001) Letzter Zugriff 20.08.2009

Kurth H (1968) Chemische Unkrautbekämpfung, 3. Aufl. VEB Gustav Fischer Verlag, Jena

Landesamt für Wasserwirtschaft (1986) Beseitigung von Ölschlamm nach einem Tankerunfall. Mitt aus dem Niedersächsischen Landesamt für Wasserwirtschaft, Heft 1 
Malkomes H.-P (2005) Mikrobiologisch-ökotoxikologische Bodenuntersuchungen von zwei im Pflanzenschutz eingesetzten Ö1-Präparaten. Umweltwiss Schadst Forsch (17):79-84

Musat F (2005) Physiological investigation of aerobic petroleum degradation in marine sediment microcosmos. Dissertation Fachbereich Biologie/Chemie Universität Bremen, Bremen

Oebius HU (2004) Physikalische Grundlagen mechanischer Bekämpfung von Flüssigchemikalien auf und in Gewässern. Koblenz. Nachdruck aus Spill Science \& Technology Bulletin 5:177-289, Copyright (1999) with permission of Elsevier
Oehlmann J, Markert B (1999) Ökotoxikologie - Ökosystemare Ansätze und Methoden. ecomed verlagsgesellschaft, Landsberg/ Lech

Riis V, Miethe D, Babel W (1998) Grenzen der Sanierbarkeit von Mineralölschäden. altlastenspectrum 4:214-218

Sächsisches Landesamt für Umwelt und Geologie (Hrsg) (2003) Freistaat Sachsen Handbuch zur Altlastenhandhabung, Bd. 7, Detailuntersuchung (Text und Anlagen), Dresden

Schneider R (2003) Das Hochwasser im August 2002. Erfahrungen der kreisfreien Stadt Dessau. Berufsfeuerwehr Dessau, Dessau 